\title{
A functional analysis of collateral behavior under differential-reinforcement-of-low-rate schedules
}

\author{
NANCY S. HEMMES \\ Queens College of the City University of New York, Flushing, New York 11367 \\ and \\ DAVID A. ECKERMAN and HILLEL J. RUBINSKY \\ University of North Carolina, Chapel Hill, North Carolina 27514
}

\begin{abstract}
The collateral behavior of pigeons under differential-reinforcement-of-low-rate (DRL) schedules was evaluated for its role in controlling DRL performance. Two of three pigeons engaged in high rates of collateral keypecking under schedules up to DRL $28 \mathrm{sec}$. Rate of collateral pecking was positively correlated with DRL efficiency. Topographical features of the collateral behavior were inconsistent with the notion that collateral behavior mediates DRL performance through response-produced stimuli. Rather, the collateral behavior appeared to interfere with the operant response, delaying it long enough to meet the DRL requirement.
\end{abstract}

When human and nonhuman subjects are exposed to schedules of reinforcement which require temporal spacing of responses, they frequently develop nonrandom patterns of collateral behavior between occurrences of the operant response. Though these collateral behaviors may merely be induced and functionally insignificant, the topographical and temporal stereotypy of these patterns has lead to speculation that they are functional in determining the temporal spacing of the operants. Such speculation is encouraged by studies which demonstrate that decrements in performance occur under differential-reinforcement-of-low-rate (DRL) schedules when subjects are prevented from engaging in collateral behavior (Laties, Weiss, \& Weiss, 1969; Richardson \& Loughead, 1974; Schwartz \& Williams, 1971). A parsimonious interpretation of these observations is that collateral behaviors facilitate DRL performance by interfering with and thereby delaying the operant response (Schwartz \& Williams, 1971). Laties et al. (1969), however, argued for a stronger interpretation, suggesting that collateral behaviors could be described as a discriminated chain of responding in which each response serves as a discriminative stimulus for the following response. In this way, the subject's own behaviors serve as a "clock," indicating the time for the operant.

Although neither of these interpretations of collateral behavior has been formalized, several means

This research was supported by Grant MH15540 from the National Institute of Mental Health to D. A. Eckerman, by NIMH Training Grant MH-14269, and by Grant 10100 from the Research Foundation of the City University of New York to N.S. Hemmes. Requests for reprints should be addressed to Nancy S. Hemmes, Department of Psychology, Queens College of CUNY, Flushing, New York 11367. of discriminating between them seem reasonable. Specifically, we shall argue that support for mediation will be demonstrated when: (1) the precision of timing under DRL schedules is functionally related to properties of the collateral behavior; (2) the collateral behavior is highly stereotyped and deviations from the stereotyped pattern lead to nonreinforcement; and (3) the sequences of collateral behavior are heterogeneous, thereby providing a set of discriminable stimuli to be used for mediation.

If mediation is not supported in these ways, then we shall argue that the functional significance of collateral behavior may be related to its ability to interfere with the operant response. One prediction of this interference hypothesis is that the degree of incompatibility between the collateral and operant behaviors should be positively related to DRL efficiency.

The preceding arguments were tested in an experiment in which the location and sequential patterns of collateral and operant keypecking of pigeons were studied in a situation where reinforcers were provided for temporally spaced operant keypecks. Much recent work with the keypeck operant has established a body of data on DRL efficiency (e.g., Richardson \& Clark, 1976), and we will compare the performance obtained to this work.

\section{METHOD}

\section{Subjects}

Three male White Carneaux pigeons were studied. They were maintained at $75 \% \pm 15 \mathrm{~g}$ of free-feeding weight.

\section{Apparatus}

The experimental enclosure measured $41 \times 37 \times 33 \mathrm{~cm}$. On one $37-\mathrm{cm}$ wall, a $25.3 \times 2.5 \mathrm{~cm}$ horizontal response strip was centered $21 \mathrm{~cm}$ above the floor. The strip, which could be 
Table 1

Correlation Coefficients Between Rate of Collateral Pecking and DRL Efficiency

\begin{tabular}{clccc}
\hline & & \multicolumn{3}{c}{ Bird } \\
\cline { 3 - 5 }$X$ & \multicolumn{1}{c}{$\mathrm{Y}$} & B173 & B175 & B190 \\
\hline Collateral Pecks per Second & SR/op & $.26(10)$ & $.57^{*}(13)$ & $.76^{* *}(22)$ \\
Collateral Pecks per Second & SR/DRL Pecks & $.33(10)$ & $.66^{* *}(13)$ & $.85^{* *}(22)$ \\
Reinforced Keys & & 1,2 & $11,12,13,14$ & $7,8,9,10$ \\
\hline
\end{tabular}

Note-Degrees of freedom are shown in parentheses. $\quad * p<.05$.

evenly transilluminated with diffuse white light, was divided into 20 independent pecking keys. Separate contingencies could be arranged for pecks to each key and responses on each key could be independently recorded. A feeder opening was centered at a height of $7.5 \mathrm{~cm}$ directly below the response strip. The chamber also contained a television camera, which permitted observation of the subject.

\section{Procedure}

The birds were shaped to peck anywhere within the response strip and were given 20 sessions in which each peck produced a reinforcer (3-sec access to grain) until 160 reinforcers were given. A DRL contingency was then programmed on part of the strip, while responses directed toward other areas of the strip were recorded but had no other programmed effect. Under the DRL schedule, pecks were reinforced if they followed the previous peck or reinforcement by an interval equal to or greater than the DRL value. Selection of the reinforced keys was based on the distribution of responses over the 20 keys during CRF. The four adjacent keys showing the highest frequency of responding were designated reinforced keys. In the case of B173, only two adjacent keys were chosen because greater than $90 \%$ of all responses were directed toward these keys. The reinforced keys programmed for each bird are listed in Table 1. The keys were numbered left to right from 1 to 20.

The birds were initially exposed to DRL $5 \mathrm{sec}$ for 15 sessions. Then the DRL requirement was increased in 2-, 3-, or 4-sec steps. The birds were exposed to each DRL value until performance attained a criterion of 10 or fewer responses on reinforced keys per reinforcement. Two birds, B175 and B190, were exposed to DRL values up to DRL $28 \mathrm{sec}$. The schedule of the third bird, B173, was discontinued at DRL $14 \mathrm{sec}$ when it failed to meet the criterion and engaged in long periods of no responding. Sessions terminated after 100 reinforcers had been delivered during the DRL 5 -sec sessions. Thereafter, only 50 reinforcers were delivered per session.

\section{RESULTS}

Two of the three birds (B175 and B190) maintained rather efficient DRL performance through the range of DRL values studied, earning roughly a third of the possible reinforcers per hour even at DRL $28 \mathrm{sec}$ (see Figure 1). The efficiency compares favorably with that found by Richardson and Clark (1976) for pigeons keypecking for food. The two efficient birds developed consistent collateral behavior. The third bird (B173) showed rapidly decreasing DRL efficiency and poor maintenance of behavior, even for DRL 10 and DRL 14. No consistent collateral behavior was seen in TV observation for this bird.

For both B175 and B190, the collateral behavior included pecking parts of the response strip not fall- ing within the reinforced keys. Since these collateral pecks were easily recorded and occurred with sufficient rate and persistence across DRL values (see Figure 1), they seemed a fruitful area of the repertoire to search for mediating or otherwise functionally related behavior.

To see if the collateral pecks were functionally related to the DRL performance, a correlation was computed between the rate of collateral pecking and DRL efficiency. Mean values of these variables for each of the last three sessions at each DRL

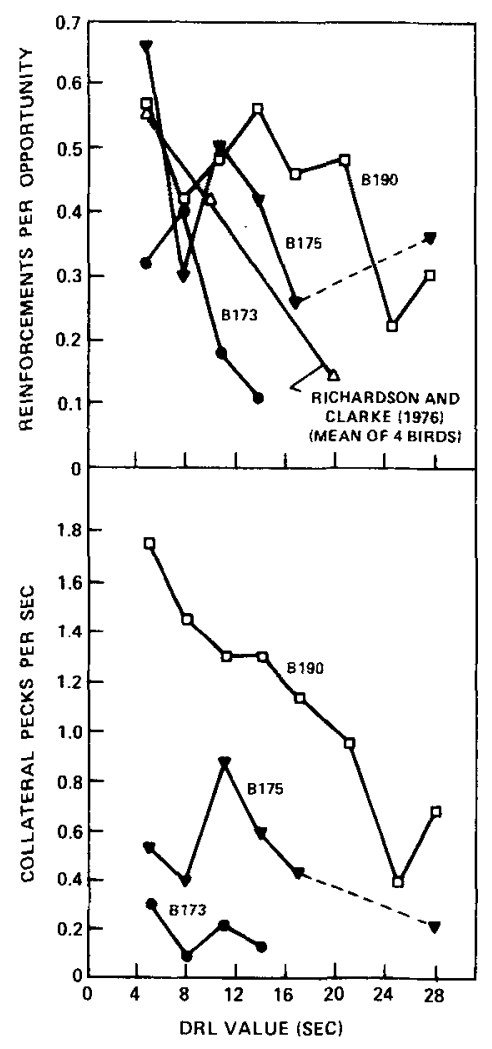

Figure 1. Top panel: Efficiency of DRL performance

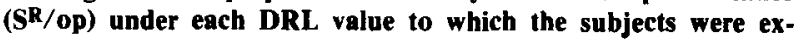
posed. Data points are based on the last 3 days under each DRL schedule. The broken line for B175 indicates missing data for DRL 20 to 26 sec. Bottom panel: Rate of collateral keypecking under all DRL values to which the subjects were exposed. Data points are based on the last 3 days under each DRL schedule. The broken line for B175 indicates missing data for DRL 20 to 26 sec. 
value from 5 to $28 \mathrm{sec}(\mathrm{B} 190)$ or 5 to $17 \mathrm{sec}(\mathrm{B} 175)$ were included in this correlation. Two types of DRL efficiency were used: reinforcers per opportunity and reinforcers per DRL response. For both measures, statistically reliable correlations were observed (Table 1). This correlation is a necessary (but not sufficient) condition to presume that the collateral pecking was functionally related to the DRL performance. To qualify as mediators of the DRL, however, these collateral pecks must be both stereotyped and heterogeneous. Therefore, these characteristics were assessed.

\section{Stereotypy and Heterogeneity of Collateral Behavior}

Stereotypy of collateral keypecking was assessed by analyzing each run of collateral responses for sequential dependencies of response location. A run was defined as all collateral pecks which intervened between two pecks on any reinforced key (Nevin \& Berryman, 1963). The first three responses in runs greater than two in length and the last three responses in runs greater than five in length were separately analyzed. The analysis consisted of listing each pattern of response location which occurred and tallying its frequency of occurrence. Runs from the last three sessions on DRL $14 \mathrm{sec}$ and DRL $28 \mathrm{sec}$ were separately analyzed for birds B175 and B190. Four tallies were made for each bird at each DRL value. These included the first three responses and the last three responses of runs terminated by reinforcement, and the first and last three responses of runs terminated by an unreinforced peck at a reinforced key. The analyses for B175 at both DRL values showed no consistent patterns-no single pattern occurred in more than $17 \%$ of the runs. Similar results were obtained for B190 under DRL $14 \mathrm{sec}$; however, when this bird was exposed to DRL $28 \mathrm{sec}$, one consistent pattern of response location emerged. This effect can be seen in Figure 2, which presents frequency histograms for response patterns in each of the four tallies described above. Bars were drawn in the histogram only when a pattern accounted for at least $2 \%$ of the patterns in a tally. An exception was made if a pattern occurred with high frequency in three tallies, but accounted for less than $2 \%$ of the patterns in the fourth tally. Figure 2 indicates that the predominant pattern for the first and last three responses in both reinforced and unreinforced runs (labeled + DRL and -DRL, respectively) was three pecks on the leftmost key-key No. 1. This pattern is designated " 111 " in the figure. The next most frequent pattern was a peck on key 2 followed by two pecks on key 1 (211). These analyses indicate that stereotypy of collateral response location was the exception rather than the rule. Further, when stereotypy was

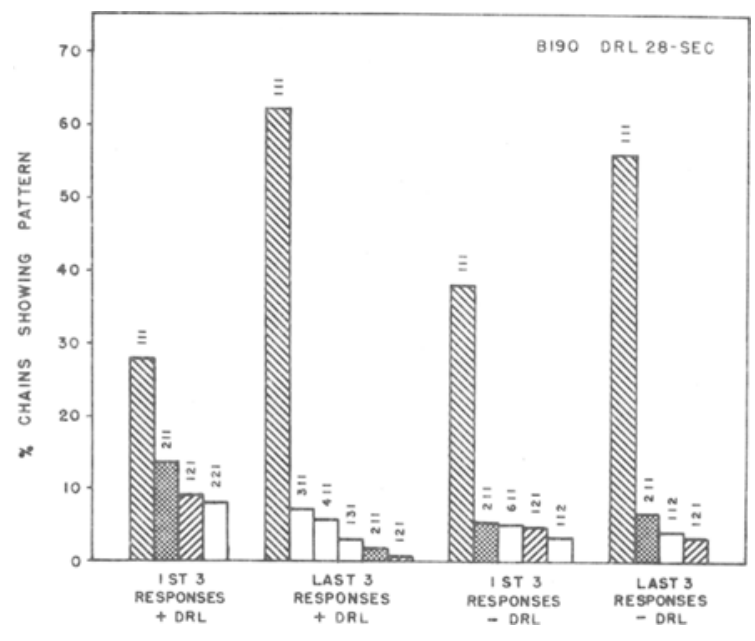

Figure 2. Percentage of collateral runs showing frequent sequential patterns for B190 under DRL 28 sec. The first and last three responses in reinforced runs (+DRL) and unreinforced runs ( - DRL) are included in this analysis, which was based on the last 3 days of exposure to DRL 28 sec.

observed, the stereotyped runs were homogeneous. These data do not suggest that the location of collateral pecks was mediating DRL performance.

\section{Incompatibility Between Collateral and DRL Pecking}

While correlation coefficients suggest that collateral behavior may be functionally related to DRL performance, no evidence was found for location of collateral pecks as a mediator of this performance, because chains of collateral pecks were not stereotyped or were homogeneous when they were stereotyped. A second possible functional relation is one of interference. That is, pecking at collateral locations may delay return to the reinforced keys and, therefore, improve DRL performance. To assess this possiblity, the location of collateral pecks was related to DRL pecks as follows: collateral responses were separated into collateral pecks preceding reinforced DRL responses and those preceding unreinforced DRL responses. For each set, the mean distance to the right and the mean distance to the left of the reinforced keys was calculated for: (1) the first response after a DRL response (this measure, called NEXT, includes both collateral and repeated DRL pecks), (2) the first response of a collateral run (called FIRST), and (3) the last response of a collateral run (called LAST). In case of runs containing only one response, FIRST and LAST would contain the same response. Figure 3 provides these measures for the last three sessions at two DRL values. The vertical axis shows mean distance to the left or right of the reinforced keys in number of keys. Responses preceding reinforced DRL responses 


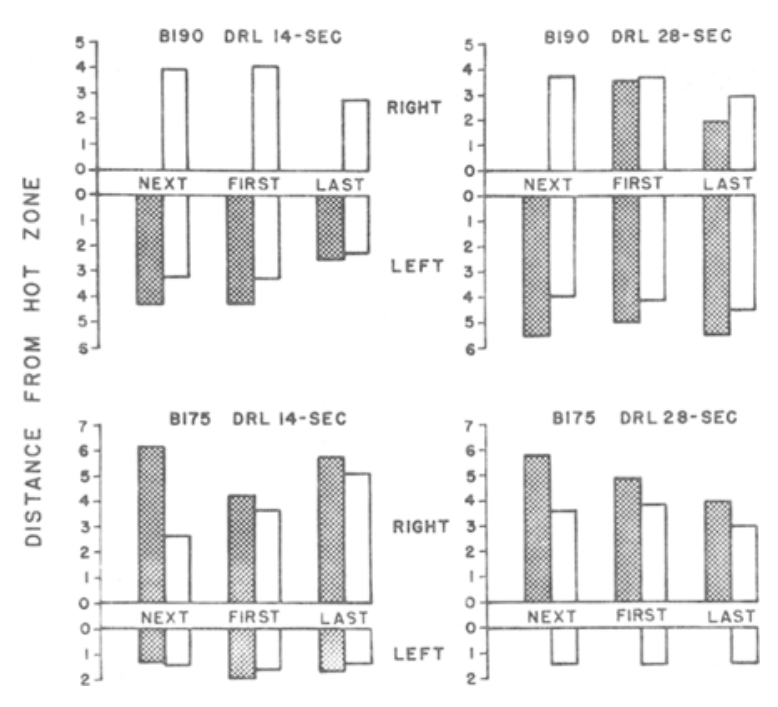

Figure 3. Mean distance (number of keys) from the reinforced keys ("hot zone") for the next, first, and last responses of collateral runs. Distances corresponding to reinforced runs are indicated by the shaded bars. Unshaded bars represent distances corresponding to unreinforced runs. The last three sessions under DRL 14 sec and DRL 28 sec were included in the analysis.

(shaded bars) were farther from the DRL keys than responses preceding unreinforced DRL responses (open bars) in 14 of 17 comparisons. Mann Whitney $U$ tests revealed that in each of these 14 cases the difference was reliable $(p \leqslant .05)$. In none of the three cases where the means were reversed was the effect reliable. It should be noted that, for B190 at DRL $14 \mathrm{sec}$ and for B175 at DRL $28 \mathrm{sec}$, relatively few collateral pecks were emitted on one of the sides of the key. Bars were included for runs preceding unreinforced DRL pecks (the LAST measure, for example, is based on 14 and on 94 pecks for B190 and $B 175$, respectively) but are not included for runs preceding reinforced DRL pecks, since these mean locations would have been based on fewer than 10 pecks. It might be noted that when sequences of pecks were considered (e.g., Figure 2), no particular sequence was differentially associated with reinforcement. The locations of the first or last response of collateral runs (Figure 3), however, was differentially associated with reinforcement. Collateral pecks preceding reinforced DRL pecks were farther from the reinforced keys than were collateral pecks preceding unreinforced DRL pecks.

\section{DISCUSSION}

By finding correlations between rate of collateral pecking and DRL efficiency, these data add to those of Schwartz and Williams (1971) and Zuriff (1969). We extend their results by demonstrating within- subject correlations (vs. between-subject correlations) between amount of collateral pecking (vs. mere presence/absence) and DRL efficiency. Other investigators (e.g., McMillan, 1969; Zeiler, 1972) have not found collateral pecking to be correlated with DRL efficiency. It is suggestive that rate of collateral pecking for these studies was very low, seemingly comparable to that shown by $\mathrm{B} 173$ of the present study, for which no relation was found with DRL efficiency. Perhaps collateral pecking must be present at greater than some criterion rate before it can assume a functional role.

A correlation with collateral behavior, of course, does not necessarily imply a causal relation. Perhaps efficient DRL peformance induces more collateral pecks or, even more plausibly, perhaps the higher reinforcer rate associated with more efficient DRL performance induces collateral pecking. The correlation, however, is suggestive of the functional relation sought by many investigators to claim that collateral behavior mediates or helps delay DRL responses. What evaluation do the present data offer these proposals?

The mediation hypothesis requires that the performance be the same from instance to instance. We found little evidence of such stereotypy, and therefore little evidence supporting mediation. Further, where stereotypy was found, a second requirement of the mediation hypothesis was not supported as the performance was found to be topographically homogeneous rather than heterogeneous. A homogeneous feature cannot provide the changing stimuli required to allow discriminative control of shifting back to the DRL keys. Of course, some other topographical characteristic of the collateral behavior might be the basis of such control (e.g., force, though force seems a less plausible dimension for this ballistic response than for others). We are looking for the veritable needle in the allegorical haystack. It might be stressed, however, that pecking was the predominant collateral behavior and that location of this behavior was related to DRL efficiency (as discussed in the next section). This seems then a very profitable area of the haystack to inspect. Special comment might be made regarding the possibility that "count" or "amount" of pecking might be the stimulus controlling return to the DRL keys. Since these are not dimensions of the behavior but of an inferred analysis of the behavior, these proposals do little to aid an experimental analysis of the putative mediating behavior. We find, therefore, no evidence for mediating behavior.

There is, however, evidence supporting the interference hypothesis. As described by Schwartz and Williams (1971), the interference hypothesis proposes that the contribution of collateral behavior to DRL performance is related to the response-constraining 
contingency imposed by DRL schedules, rather than to the temporal contingency. There is a large literature noting that, though sensitive to these contingencies, keypecking does not conform as well as other operants to procedures requiring delaying or refraining from responding to produce food (Hemmes, 1975; Richardson \& Clark, 1976; Williams \& Williams, 1969). If the collateral behavior interferes with DRL pecks, then, it might well increase DRL efficiency. In the present study, collateral pecks more distant from the reinforced keys (and, therefore, more compatible with return to the reinforced keys) were differentially associated with reinforced DRL responses. Far from being stereotyped, therefore, there seems to be a continuing dynamic in this collateral behavior, with more distant pecking being continuously shaped by higher reinforcer rates. Barrera (1974) has made a related observation. Pigeons were exposed to a procedure where pecks to a signal cancelled reinforcers which otherwise would follow the signal (negative automaintenance). While no pigeon refrained from pecking altogether, several learned to redirect some pecks toward locations other than the key. Pigeons which directed these off-key pecks farthest away showed the lowest number of pecks to the signal. The complementary observation has also been made. Several investigators have shown that when collateral responses are restricted to the area just surrounding the operandum, DRL efficiency has decreased (Glazer \& Singh, 1971; Richardson \& Loughead, 1974; Skuban \& Richardson, 1975).

The present data, therefore, add to those supporting an interference, rather than a mediating, role for collateral pecking.

\section{REFERENCES}

Barrera, F. J. Centrifugal selection of signal-directed pecking. Journal of the Experimental Analysis of Behavior, 1974, 22, 341-355.

Glazer, H., \& Singh, D. Role of collateral behavior in temporal discrimination performance and learning in rats. Journal of Experimental Psychology, 1971, 91, 78-84.
Hemmes, N. S. Pigeons' performance under differential reinforcement of low rate schedules depends upon the operant. Learning and Motivation, 1975, 6, 344-357.

Laties, V. G., Weiss, B., Clark, R. L., \& Reynolds, M. D. Overt "mediating" behavior during temporally spaced responding. Journal of the Experimental Analysis of Behavior, 1965, 8, 107-116.

Laties, V. G., Weiss, B., \& Weiss, A. B. Further observations on overt "mediating" behavior and the discrimination of time. Journal of the Experimental Analysis of Behavior, 1969, 12, 43-57.

McMillan, D. E. Reinforcement contingencies maintaining collateral responding under a DRL schedule. Journal of the Experimental A nalysis of Behavior, 1969, 12, 413-422.

Nevin, J. A., \& Berryman, R. A note on chaining and temporal discrimination. Journal of the Experimental Analysis of Behavior, 1963, 6, 109-113.

Richardson, W. K., \& Clark, D. B. A comparison of the keypeck and treadle-press operants in the pigeon: Differentialreinforcment-of-low-rate schedule of reinforcement. Journal of the Experimental Analysis of Behavior, 1976, 26, 237-256.

Richardson, W. K., \& Loughead, T. E. The effect of physical restraint on behavior under the differential-reinforcement-oflow-rate schedule. Journal of the Experimental Analysis of Behavior, 1974, 21, 455-461.

Schwartz, B., \& Williams, D. R. Discrete-trials spaced responding in the pigeon: The dependence of efficient performance on the availability of a stimulus for collateral pecking. Journal of the Experimental Analysis of Behavior, 1971, 16, 155-160.

Skuban, W. E., \& Richardson, W. K. The effect of the size of the test environment on behavior under two temporally defined schedules. Journal of the Experimental Analysis of Behavior, 1975, 23, 271-275.

Williams, D. R., \& Williams, H. Auto-maintenance in the pigeon: Sustained pecking despite contingent non-reinforcement. Journal of the Experimental Analysis of Behavior, 1969, 12, $511-520$.

ZEILER, M. D. Reinforcement of spaced responding in a simultaneous discrimination. Journal of the Experimental Analysis of Behavior, 1972, 18, 443-451.

ZuRIFF, G. E. Collateral responding during differential reinforcement of low rates. Journal of the Experimental Analysis of Behavior, 1969, 12, 971-976.

(Received for publication August 3, 1978; revision accepted October 31,1978 .) 